\title{
Results of Fertility-Sparing Surgery for Expansile and Infiltrative Mucinous Ovarian Cancers
}

\author{
Sebastien Gouy, ${ }^{a}$ Marine Saidani, ${ }^{a}$ Amandine Maulard, ${ }^{a}$ Slim Bach-Hamba, ${ }^{b}$ Enrica Bentivegna, ${ }^{a}$ Alexandra Leary, \\ Patricia Pautier, ${ }^{b}$ Mojgan Devouassoux-Shisheboran, ${ }^{e}$ Catherine Genestie, ${ }^{c}$ Philippe Morice ${ }^{a, f, g}$ \\ Departments of a Gynecologic Surgery, ${ }^{b}$ Medical Oncology, and ${ }^{\mathrm{c} P a t h o l o g y, ~ G u s t a v e ~ R o u s s y, ~ V i l l e j u i f, ~ F r a n c e ; ~}{ }^{\mathrm{d} I N S E R M ~ U 981, ~ G u s t a v e ~ R o u s s y, ~}$ \\ Villejuif, France; ${ }^{\mathrm{e} D e p a r t m e n t}$ of Pathology, Hospices Civils de Lyon, Lyon, France; ${ }^{\mathrm{f} U n i t}$ INSERM 1030, Villejuif, France; ${ }^{\mathrm{g}}$ University Paris Sud, \\ Paris, France \\ Disclosures of potential conflicts of interest may be found at the end of this article.
}

Key Words. Conservative treatment • Expansile • Infiltrative • Mucinous cancer • Ovary

\section{ABSTRACT}

Background. No series had been reported focusing on the results of fertility-sparing surgery in stage I mucinous ovarian cancers according to histotype (infiltrative vs. expansile). Investigating such outcomes was the aim of the present study.

Materials and Methods. The present study was a retrospective analysis of patients treated conservatively with preservation of the uterus and contralateral ovary from 1976 to 2016. The pathology of the tumors was reviewed by two expert pathologists according to the 2014 World Health Organization (WHO) classification criteria. Oncologic and fertility results were analyzed.

Results. Twenty-one patients fulfilled the inclusion criteria, twelve with expansile and nine with infiltrative cancer. All patients had a unilateral tumor and underwent unilateral salpingo-oophorectomy in one-step $(n=6)$ or two-step $(n=15)$ surgeries. All but one had complete peritoneal staging surgery based on cytology, omentectomy, and random peritoneal biopsies. Ten had nodal staging surgery. The International Federation of Gynecology and Obstetrics stages were IA $(n=9)$, IC1 $(n=6)$, and IC2 $(n=6)$; the nuclear grades were grade $1(n=9)$, grade $2(n=5)$, and grade $3(n=1)$. Two patients recurred (one expansile and one infiltrative type) 19 and 160 months after surgery, respectively. One stage IA, nuclear grade 2 expansile tumor recurred on the spared ovary; the patient remains alive. The other stage IA infiltrative tumor recurred as peritoneal spread; the patient is alive with disease. Six patients became pregnant; four with expansile tumors and two with infiltrative tumors.

Conclusion. The type of mucinous cancer has no impact on the oncologic outcome in this series of patients treated conservatively. Fertility-sparing surgery should be considered for earlystage infiltrative-type tumors. The Oncologist 2018; 23:324-327

Implications for Practice: According to the most recently updated World Health Organization classification guidelines, mucinous cancers should be classified as either expansile or infiltrative. The infiltrative type has a poorer prognosis, but there are no data about the safety of fertility-sparing surgery (FSS) in this context. A collection of 21 cases reviewed by two expert pathologists this study is the first devoted to the conservative treatment of mucinous tumors according to both subtypes. The key result was that the type of mucinous cancer has no impact on the oncologic outcome; thus, FSS may be considered in both subtypes.

\section{INTRODUCTION}

Mucinous ovarian carcinomas (mOCs) represent a complex group of epithelial tumors. Over the last 4 decades, different classification systems have been used to describe these tumors $[1,2]$. In 1973, Hart and Norris reported a category of what they termed "noninvasive mucinous carcinoma" with stratification of more than three cell layers that showed severe nuclear atypia [3]. In 2000, Lee and Scully described the expansile and infiltrative types of $\mathrm{mOC}$ based on morphological and prognostic differences [4]. In 2014, in order to standardize the pathological reporting of gynecological tumors, World Health Organization (WHO) guidelines proposed classifying the primary mucinous cancers in these two groups based on their growth patterns, calling them expansile- and infiltrative-type tumors [1]. The prognosis for expansile-type tumors is better than that for infiltrative-type tumors [4].

Two characteristics were observed in mOCs compared with other subtypes of epithelial cancers. First, mOCs are diagnosed more frequently at an early stage; second, they affect younger patients. Accordingly, when we evaluate the use of fertilitysparing surgery (FSS) in epithelial ovarian cancer treatment, $\mathrm{mOC}$ is the most frequent subtype for which FSS is considered $[5,6]$. But no studies have investigated the outcomes of FSS in 
Table 1. Characteristics of patients with mucinous ovarian cancers according to histotype

\begin{tabular}{|c|c|c|}
\hline Characteristics & $\begin{array}{l}\text { Expansile } \\
(n=12)\end{array}$ & $\begin{array}{l}\text { Infiltrative } \\
(n=9)\end{array}$ \\
\hline Median age, years (range) & $24(17-42)$ & $27(17-41)$ \\
\hline Body mass index, $\mathrm{kg} / \mathrm{m}^{2}$ (range) & $22(21-31)$ & $25(19-34)$ \\
\hline \multicolumn{3}{|l|}{ Previous history } \\
\hline Borderline $e^{a}$ & 0 & 0 \\
\hline Cancer $^{\mathrm{b}}$ & 0 & 1 \\
\hline Previous appendectomy & 4 & 2 \\
\hline Median tumor diameter, $\mathrm{cm}$ (range) & $15(7-26)$ & $16(4-23)$ \\
\hline \multicolumn{3}{|l|}{ Peritoneal staging } \\
\hline Cytology & 12 & 9 \\
\hline Peritoneal biopsies & 12 & 9 \\
\hline Omentectomy or omental biopsies & 11 & 9 \\
\hline Appendectomy & 12 & 8 \\
\hline Complete peritoneal staging ${ }^{c}$ & 11 & 9 \\
\hline \multicolumn{3}{|l|}{ Lymph nodes staging } \\
\hline Pelvic and para-aortic & 3 & 7 \\
\hline $\begin{array}{l}\text { Median number of nodes } \\
\text { removed (range) }\end{array}$ & $9(1-29)$ & $14.5(2-42)$ \\
\hline $\begin{array}{l}\text { One-step surgery (ovarian disease } \\
\text { and staging procedures) }\end{array}$ & 5 & 1 \\
\hline Laparotomy & 3 & 1 \\
\hline Laparoscopy & 1 & 0 \\
\hline Unknown & 1 & \\
\hline Two-step surgery & 7 & 8 \\
\hline Median delay in months (range) & $2(0.5-9)$ & $2(1.5-3)$ \\
\hline Laparotomy only & 3 & 3 \\
\hline Laparoscopy only & 1 & 1 \\
\hline Combined modalities & 3 & 2 \\
\hline Unknown & 0 & 2 \\
\hline \multicolumn{3}{|l|}{ FIGO stage } \\
\hline IA & 5 & 4 \\
\hline IC1 & 4 & 2 \\
\hline IC2 & 3 & 3 \\
\hline IC3 & 0 & 0 \\
\hline \multicolumn{3}{|l|}{ Grade } \\
\hline Grade 1 & 5 & 4 \\
\hline Grade 2 & 4 & 1 \\
\hline Grade 3 & 0 & 1 \\
\hline \multicolumn{3}{|c|}{$\begin{array}{l}{ }^{\mathrm{a}} \text { Mucinous } \\
{ }^{\mathrm{b}} \text { Breast cancer } \\
\text { cSurgical procedures, including those performed during initial and } \\
\text { restaging surgery (if restaging surgery was performed). } \\
\text { Abbreviation: FIGO, International Federation of Gynecology and } \\
\text { Obstetrics. }\end{array}$} \\
\hline
\end{tabular}

mOCs according to their subtypes. However, this question is relevant in order to determine whether one subtype is intrinsically more aggressive than the other. In the three papers that address the pathological description of different mucinous diseases, conservative surgeries are briefly described, but no or very few specific outcomes are reported $[4,7,8]$. Thus, the aim of the current analysis was to investigate the outcomes of conservative $\mathrm{FSS}$ in $\mathrm{mOC}$ patients according to tumor histotype (i.e., expansile vs. infiltrative).

\section{Patients}

We retrospectively identified patients with $\mathrm{mOC}$ who were referred to or treated at our institution between 1976 and 2016. The inclusion criteria were as follows. First, two expert pathologists (C.G. and M.D.S.) had to be able to perform a review of the tumor pathology according to the 2014 WHO classification criteria. If the initial ovarian tumor was not available for pathologic review (as for the oldest cases and for cases treated outside our institution), the patients were excluded. Only patients having a mucinous carcinoma were included. Patients having a borderline mucinous tumor, an intraepithelial carcinoma, or a stromal invasion $<5 \mathrm{~mm}$ in the greatest linear dimension (defining a microinvasion) were not analyzed in the current series. Second, the tumor had to be macroscopic stage I disease, with an absence of extraovarian disease during the surgical exploration. Third, there had to be sufficient surgical and clinical data available so that we could determine the exact surgical procedures that were carried out and their outcomes. Fourth, FSS after one- or two-step surgery (in cases with restaging surgery) was defined as the preservation of the uterus and the ovary contralateral to the initial tumor with the aim of fertility preservation.

"Complete" peritoneal surgical staging was defined when peritoneal cytology, multiple peritoneal biopsies, omentectomy, or omental biopsies were performed. In some cases, ovarian tumor surgery and staging (if it was done) was performed in a one-step surgery in which the malignancy was diagnosed during the surgery using frozen section analysis. In other cases, the staging surgery was performed using a two-step surgery (restaging surgery). These surgeries were carried out by laparoscopic or laparotomic approaches based on the decision made by the surgical team. The tumors were typed as expansile or infiltrative according to the 2014 WHO classification criteria [1]. There is a consensus that the grading system used for serous cancers should not be used for mucinous subtypes. The 2014 WHO classification does not have recommendations about the grading system that should be used for mOCs. In the present series, for expansile subtype, we used the nuclear grading system that was recommended by Rodríguez and Prat [7]. The 2014 International Federation of Gynecology and Obstetrics (FIGO) staging system was used; it includes three new classes of stage IC disease [9]. The surgical and clinical outcomes of FSS were determined and compared for the two histotypes.

\section{Patient Characteristics ANd OnCological AND FERTILITY RESUlts}

We reviewed 114 cases. Forty-six were excluded because the macroscopic disease severity was greater than stage I ( $n=26$ cases: 2 stage II, 18 stage III, 4 stage IV, and 2 unknown) or because of the lack of accessibility for a pathologic review $(n=20)$. The final analysis included 68 cases: 29 expansile and 39 infiltrative mOC types. Twenty-one of these patients underwent FSS (12 expansile and 9 infiltrative). Table 1 shows the characteristics of the patients. All had unilateral tumors and underwent unilateral salpingo-oophorectomy in one-step 
Table 2. Fertility outcomes of patients with mucinous ovarian cancers according to histotype

\begin{tabular}{lll}
\hline Characteristics & $\begin{array}{l}\text { Expansile } \\
(\boldsymbol{n}=\mathbf{1 2})\end{array}$ & $\begin{array}{l}\text { Infiltrative } \\
(\boldsymbol{n}=\mathbf{9})\end{array}$ \\
\hline Median age, years (range) & $22.5(20-27)$ & $27.5(26-29)$ \\
Previous history of infertility & 0 & 0 \\
Nulliparous & & \\
$\quad$ Yes & 2 & 2 \\
No & 2 & 0 \\
Unknown & 8 & 7 \\
Pregnancy & & \\
$\quad$ In nulliparous patients & 2 & 2 \\
$\quad$ Spontaneous & 4 & 1 \\
$\quad$ Medically assisted & 0 & 1 \\
\hline
\end{tabular}

( $n=6$ ) or two-step $(n=15)$ surgeries. All but one patient had complete peritoneal staging surgery based on cytology, omentectomy, and random peritoneal biopsies; the exception was a patient who had an expansile-type tumor. Ten patients had nodal staging surgery and were free of disease. Four patients who initially underwent cystectomy had salpingo-oophorectomy (with staging surgery) during the restaging procedure. We do not have the details of the number of uterine curettages used to stage the disease in these patients.

The FIGO stages were IA $(n=9)$, IC1 $(n=6)$, and IC2 $(n=6)$. Table 1 shows the distribution of the two histotypes. Twelve patients received adjuvant chemotherapy, with platinum-based regimens used in ten.

After a median time of follow-up of 46 (range, 1-179) months, two patients recurred, one who had an expansile-type tumor and one with an infiltrative type. The first patient had a stage IA expansile-type tumor that was nuclear grade 2 . She underwent complete peritoneal and nodal staging surgery (negative nodes) via a two-step procedure and received adjuvant chemotherapy. She had a spontaneous pregnancy. Nineteen months after the end of the initial treatment, she had a contralateral ovarian recurrence treated with radical surgery followed by chemotherapy. She is currently alive (75 months after the recurrent disease).

The second patient had an initial stage IA infiltrativesubtype tumor. She underwent exclusive surgery (without adjuvant treatment) that included peritoneal staging and subsequently had a twin pregnancy via oocyte donation. She had recurrence with a mucinous borderline tumor on the contralateral ovary 44 months after her initial treatment and underwent a radical surgery and a nodal staging surgery. She showed recurrence 116 months later with massive peritoneal spread that was treated by chemotherapy and salvage surgery. At the time of this report, 168 months after the diagnosis of her initial tumor, she is alive with progressive disease.

Table 2 shows the fertility results for the 21 patients. There were six pregnancies in four patients with expansile-type tumors and in two patients with infiltrative-type tumors. Of these pregnancies, five were spontaneous; two patients had pregnancies before recurrent disease.

\section{DISCUSSION}

These data add to the current knowledge regarding $\mathrm{mOC}$ treatment. Issues related to FSS in epithelial ovarian cancer were reviewed recently [5]. That review suggested that FSS is safe for stage I disease in patients with an excellent prognosis, but it should be questioned for stage I disease in patients with the poorest prognosis (including those with grade 3 or stage IC2/ IC3 disease), who are conventionally treated with adjuvant chemotherapy after the initial surgery [5]. Nevertheless, some experts disagree with this, asking whether the poor prognosis is due to the preservation of the ovary itself (raising a question about the safety of FFS) or whether it is due to the natural history of the grade 3 and/or stage IC2/3 disease and is unrelated to ovary preservation. If the latter is correct, FSS could be proposed for stage I high-risk patients.

Aside from hypothetical considerations, it is important to address how to best manage mOC patients clinically in 2017 and going forward. The $\mathrm{mOC}$ cases studied here are particularly relevant because $\mathrm{mOC}$ is the most frequent histologic subtype in young patients affected by epithelial ovarian cancer [5]. Thus, it is critical that clinicians understand the indications and limits of FSS. In a previous FSS series, the subtypes were mixed [5]. Only one paper was specifically devoted to the outcomes of FSS in $\mathrm{mOC}$, but it did not distinguish between expansile and infiltrative tumors [10]. However, the 2014 WHO classification requires $\mathrm{mOCs}$ to be classified as one of these two types [1]. The current study is the first to specifically examine the outcomes of FSS according to tumor histotype (i.e., expansile vs. infiltrative).

mOCs are a complex group of tumors. They are frequently grouped together as being the same malignant ovarian disease, but they show histology that falls somewhere in the range of benign cystadenomas, borderline patterns, and invasive disease. Accordingly, the quality of the samples and the expertise of the pathologist are crucial for correct identification and characterization of these tumors. In the current series, we wanted to have a very accurate analysis in terms of pathological diagnosis in order to determine the safety of FSS in these patients. Toward this end, two pathologists who are experts in gynecological cancer reviewed all of the pathology slides.

The expansile subtype has a better prognosis than the infiltrative subtype $[2,4,7,11]$. Infiltrative lesions have poorly differentiated morphology and more frequently show high nuclear grade. Therefore, FSS is less frequently proposed for early-stage infiltrative-type lesions. Although the recent series by Muyldermans et al. compared expansile and infiltrative lesions, but no information was provided regarding the number and outcomes of patients treated with FSS [11].

Previous papers about the use of conservative approaches are pathological analyses that give very few details about the outcomes of patients. The series reported by Riopel et al. noted nine salpingo-oophorectomies and one cystectomy, but specific outcomes were not reported [8]. Lee and Scully reported three patients with expansiletype mOCs who underwent salpingo-oophorectomy with no recurrence [4]. The two patients with infiltrative subtypes who were treated conservatively were disease-free 5 and 12 years later. The third patient had a mixed intraepithelial borderline tumor that was a grade 1 expansile lesion with foci of infiltrative microcarcinomas, the largest measuring $4 \times$ 
$1.5 \mathrm{~mm}$. Her disease recurred 3 years later as peritoneal carcinoma, and she died from the disease [4]. Rodríguez and Prat described seven patients with expansile-type tumors who underwent unilateral salpingo-oophorectomy; the specific outcomes of these seven cases were not reported [7]. However, four patients with infiltrative-type tumors underwent similar surgeries and were disease-free at a mean follow-up of 5.6 years (range, 1-15 years). One patient who was treated conservatively had a preoperative rupture of a macroscopic stage I infiltrative carcinoma (at least stage IC2). She died from peritoneal recurrence shortly after her initial management. Another patient treated using unilateral salpingooophorectomy had a stage IC1, expansile-type tumor with several foci showing infiltrative stromal microinvasion, with the largest being $1.5 \mathrm{~mm}^{2}$. Her disease recurred 5 years later, and she died from the disease (specifically from bone and lung metastasis) [7].

Ludwick et al. reported four cases of aggressive expansiletype disease. In this series, three young patients were treated using unilateral salpingo-oophorectomy and showed recurrence, with two dying from the disease [12]. The first patient had a stage I expansile tumor with intraepithelial carcinoma and microinvasive carcinoma; the second one had a stage I tumor that was an expansile type with intraepithelial carcinoma; and the third patient had a tumor with intraepithelial and microinvasive disease [12].

It is evident that these reports aimed mainly to describe the pathological entities, but taken together, they are fragmentary and are difficult to translate into clinical practice. They do not specifically address the safety of FSS in MOC. Our main finding was that the rate of recurrence, which is fortunately low in the patients who were adequately staged with stage I mOC, was not increased for infiltrative-type lesions. This was somewhat surprising, as these tumors are considered more aggressive, compared with expansile subtype. Furthermore, the only recurrence observed for an infiltrative lesion did not seem to be related to the initial preservation of the ovary itself, because the recurrence occurred very late (160 months) after the initial conservative surgery. In this interval, the woman had a first recurrence in the form of a mucinous borderline tumor that was treated by removing the retained ovary. So, even if this is considered a recurrence after an initial conservative treatment of an infiltrative-type lesion, the data should be interpreted with caution.

\section{CONCLUSION}

In other epithelial cancers, grade 3 disease is considered by different teams to represent an "oncological limit" in terms of recurrence rate and safety for the use of FSS [5]. Our results suggest that in $\mathrm{MOC}$, for which the grading system is not similar to the scale used for serous tumors, the presence of an infiltrative tumor should not be considered to rule out FSS. Finally, the type of $\mathrm{mOC}$ does not seem to impact the oncologic outcomes in stage I mOCs, and FSS should be considered in early-stage infiltrative-type tumors using the same criteria as for expansiletype tumors. Regardless of histotype, this series did not have any cases of stage IC3 disease, so the safety of FSS for IC3 disease could not be evaluated. This suggests that as for other epithelial cancers, this disease stage should be considered the limit of the indication for FSS.

\section{ACKNOWLEDGMENTS}

S.B.H. was the recipient of a DUERTECC/EURONCO grant (Diplôme Universitaire Européen de Recherche Translationnelle Et Clinique en Cancérologie).

\section{AuTHor ConTributions \\ Conception/design: Sebastien Gouy, Philippe Morice \\ Provision of study material or patients: Sebastien Gouy, Marine Saidani, Mojgan Devouassoux-Shisheboran, Catherine Genestie, Philippe Morice \\ Collection and/or assembly of data: Sebastien Gouy, Marine Saidani, Philippe Morice \\ Data analysis and interpretation: Sebastien Gouy, Marine Saidani, Amandine Maulard, Slim Bach-Hamba, Enrica Bentivegna, Alexandra Leary, Patricia Pautier, Mojgan Devouassoux-Shisheboran, Catherine Genestie, Philippe Morice Manuscript writing: Sebastien Gouy, Philippe Morice \\ Final approval of manuscript: Sebastien Gouy, Marine Saidani, Amandine Maulard, Slim Bach-Hamba, Enrica Bentivegna, Alexandra Leary, Patricia Pautier, Mojgan Devouassoux-Shisheboran, Catherine Genestie, Philippe Morice}

\section{DisclosuRES}

Sebastien Gouy: Roche (C/A); Alexandra Leary: GamaMabs Pharma (C/A), AstraZeneca, Gristone, Clovis (SAB), GamaMabs Pharma, AstraZeneca, Roche, Merus, Clovis (RF). The other authors indicated no financial relationships.

(C/A) Consulting/advisory relationship; (RF) Research funding; (E) Employment; (ET) Expert testimony; (H) Honoraria received; (OI) Ownership interests; (IP) Intellectual property rights/ inventor/patent holder; (SAB) Scientific advisory board

\section{REFERENCES}

1. Kurman RJ, Carcangiu ML, Herrington CS et al. WHO Classification of tumours of female reproductive organs. 4th ed. Lyon, France: IARC, 2014: Chapter I.

2. Hauptmann $S$, Friedrich $K$, Redline R et al. Ovarian borderline tumors in the 2014 WHO classification: Evolving concepts and diagnostic criteria. Virchows Arch 2017;470:125-142.

3. Hart WR, Norris HJ. Borderline and malignant mucinous tumors of the ovary. Histologic criteria and clinical behavior. Cancer 1973;31:10311045.

4. Lee KR, Scully RE. Mucinous tumors of the ovary: A clinicopathologic study of 196 borderline tumors (of intestinal type) and carcinomas, including an evaluation of 11 cases with 'pseudomyxoma peritonei'. Am J Surg Pathol 2000;24: 1447-1464.
5. Bentivegna E, Gouy S, Maulard A et al. Fertilitysparing surgery in epithelial ovarian cancer: A systematic review of oncological issues. Ann Oncol 2016:27:1994-2004.

6. Colombo N, Parma G, Lapresa MT et al. Role of conservative surgery in ovarian cancer: The European experience. Int J Gynecol Cancer 2005;15(suppl 3):206-211.

7. Rodríguez IM, Prat J. Mucinous tumors of the ovary: A clinicopathologic analysis of 75 borderline tumors (of intestinal type) and carcinomas. Am J Surg Pathol 2002;26:139-152.

8. Riopel MA, Ronnett BM, Kurman RJ. Evaluation of diagnostic criteria and behavior of ovarian intestinal-type mucinous tumors: atypical proliferative (borderline) tumors and intraepithelial, microinvasive, invasive, and metastatic carcinomas. Am J Surg Pathol 1999;23:617-635.
9. Prat J; FIGO Committee on Gynecologic Oncology. Staging classification for cancer of the ovary, fallopian tube, and peritoneum. Int J Gynaecol Obstet 2014;124:1-5.

10. Lee JY, Jo YR, Kim TH et al. Safety of fertilitysparing surgery in primary mucinous carcinoma of the ovary. Cancer Res Treat 2015;47:290-297.

11. Muyldermans K, Moerman P, Amant F et al. Primary invasive mucinous ovarian carcinoma of the intestinal type: Importance of the expansile versus infiltrative type in predicting recurrence and lymph node metastases. Eur J Cancer 2013; 49:1600-1608.

12. Ludwick C, Gilks CB, Miller D et al. Aggressive behavior of stage I ovarian mucinous tumors lacking extensive infiltrative invasion: A report of four cases and review of the literature. Int J Gynecol Pathol 2005;24:205-217. 